\title{
Review
}

\section{The Future of Surgical Treatments for Parkinson's Disease}

\author{
Darrin J. Lee $\mathrm{e}^{\mathrm{a}}$ and Andres M. Lozano ${ }^{\mathrm{b}, *}$ \\ ${ }^{a}$ Department of Neurological Surgery, University of Southern California, Los Angeles, CA, USA \\ ${ }^{\mathrm{b}}$ Department of Neurological Surgery, University of Toronto, Toronto, ON, Canada
}

Accepted 10 October 2018

\begin{abstract}
The surgical treatment of Parkinson's disease has made significant progress over the past 70 years; however, its scope of effectiveness remains limited to motor symptoms like bradykinesia, rigidity, tremor and medication-induced dyskinesias. The field of surgery initially developed from lesioning procedures and then transitioned largely to deep brain stimulation due to its properties of adaptability and reversibility. Interestingly, there has been a renewed interest in lesioning procedures secondary to the introduction of focused ultrasound, a non-invasive technology. Despite the various current therapies' effectiveness, there is a significant need for developing treatments to modify the disease process itself. To date, gene therapy, immunotherapy, and cell transplantation trials have had both promising and disappointing results. Newer techniques being developed (optogenetics, magnetogenetics, and sonogenetics) are exciting possibilities for the future. Here, we examine and speculate on novel potential surgical treatments for Parkinson's disease.
\end{abstract}

Keywords: Parkinson's disease, surgery, neuromodulation

Over the last 70 years, the surgical treatment of Parkinson's disease (PD) has made significant advancements in techniques and technology as well as facilitated a better understanding of the pathophysiology of the disease. Since the 1940s, over 8,000 articles have been published on the surgical management of PD [1]. The initial surgical management of PD was limited to lesioning procedures; over time, however, particularly in the last 25 years, deep brain stimulation has become the predominant therapy. Current surgical indications for PD include reducing motor fluctuations, "off time," dyskinesias, tremor, and levodopa-responsive symptoms, although there has been significant interest in expanding the scope of these surgical treatments.

${ }^{*}$ Correspondence to: Andres M. Lozano, MD, PhD, 399 Bathurst Street, Toronto, MST 258, ON, Canada. Tel.: +1 416603 6200; E-mail: Andres.Lozano@uhnresearch.ca.

\section{DEEP BRAIN STIMULATION ANATOMY AND TECHNOLOGY}

There have been a number of recent advances in the field of neuromodulation for PD, including exploring new anatomical targets, improving technology, and exploring novel therapies. Since the early pallidotomies, the basal ganglia has been targeted to treat the motor symptoms of PD [2, 3]. Currently, the internal segment of the pallidum (GPi) and the subthalamic nucleus (STN) are the predominant surgical targets, with the thalamus being targeted in very select cases [4]. Both lesions and deep brain stimulation (DBS) at these targets can be very effective in improving a number of motor symptoms. Surgery is however less effective at treating other symptoms including gait disturbances, freezing, balance, speech, and cognition [5]. Consequently, in addition to the GPI and STN, there has been interest in exploring other targets 
to improve these other symptoms, such as the pedunculopontine nucleus (gait) [6] and nucleus basalis of Meynert (cognition) [7].

Surgical targeting of all the desired nuclei has improved with better imaging techniques, including high field strength magnetic resonance, tractography and functional imaging, and more detailed understanding of the abnormal and surrounding electrophysiology. These advances have helped to optimize surgical accuracy and reduce adverse effects. DBS technology has also significantly developed, particularly within the last ten years. There have been substantial developments in the power source for the devices. The improved battery life and the advent of rechargeable batteries have enabled patients to have fewer subsequent surgeries. Multiple stimulation parameters can be programmed for a particular patient as well as interleaving stimulation paradigms. New directional DBS electrodes are also now available. With directional leads, it is possible to shape and/or steer current to stimulate certain regions while avoiding the unwanted activation of adjacent areas [8, 9]. These innovations have allowed for more personalized and patient-specific treatments.

New implanted devices also have the capacity to record and store electrophysiological data such as local field potentials from the implanted electrode contacts. This capability to record and store the patient's electrophysiological data on an ongoing basis enables physicians to better understand the disease process. For example, there is evidence that aberrant electrophysiology such as pathological oscillations and coupling may serve as potential biomarkers for neuromodulation to target [10]. Furthermore, current DBS systems are on continuously but patients do not suffer from their symptoms continuously throughout the day. Continuous DBS may disrupt not only pathologic neural activity that produces symptoms but also normal physiological activity when it is present. Utilizing an electrographic biomarker in real-time for adaptive or "closed loop" stimulation may help to refine and optimize stimulation treatments [11].

\section{NON-INVASIVE DEEP BRAIN STIMULATION}

As DBS currently requires open surgery, there has been increasing interest in finding less invasive neuromodulation techniques. More recently, there has been a renewed interest in lesioning procedures through focused ultrasound (Fig. 1). While it is currently approved for essential tremor, there is increasing interest in treating PD with subthalamotomies [12] or pallidotomies [13] via a less invasive treatment modality. While this therapy does not require an implanted device or successive operations for battery exchanges, it still lacks from the adaptability of DBS; and due to the perceived high risk of adverse effects associated with bilateral lesions in homologous brain targets, it largely remains a unilateral procedure in a bilateral disease. Transcranial magnetic stimulation (TMS) has also been pursued as another non-invasive neuromodulation strategy; however, the results have been controversial and, so far, this has not become mainstream. Moreover, TMS predominantly effects superficial cortical brain tissue but is unable to modulate deep brain targets and circuitry, such as the basal ganglia.

A recent study by Grossman et al. demonstrated that non-invasive stimulation might be possible by utilizing offsetting non-physiologic high frequency stimulation (i.e., $2.00 \mathrm{kHz}$ and $2.01 \mathrm{kHZ}$ ) to create focal subcortical low frequency stimulation at a specific desired location [14]. Interestingly, they report being able to specifically target stimulation to the hippocampus without stimulating the overlying cortical tissue. With this type of technology, it would potentially be possible to decrease complications from open surgery (such as infections, lead migration/ fracture, etc.) and obviate the need for internalized batteries. This approach could also be used as a noninvasive stimulation-mapping tool to guide lesional treatments.

\section{DISEASE MODIFICATION}

While DBS is effective in treating motor symptoms, it does not alter the course of the disease. Thus, disease-modifying treatments are urgently needed. In an attempt to treat the underlying neurodegenerative process, there has been PD gene therapy clinical trials aimed at altering enzymes and neurotrophic factors within the basal ganglia. To date, these trials have demonstrated the capability to induce directed neuronal protein expression safely; however, the clinical efficacy has been less promising [15].

Immunotherapy has also been posited as a potential disease-modifying treatment modality. Both animal models and human clinical trials have primarily utilized antibodies to target misfolded $\alpha$-synuclein, the primary protein component in Lewy bodies. It is 


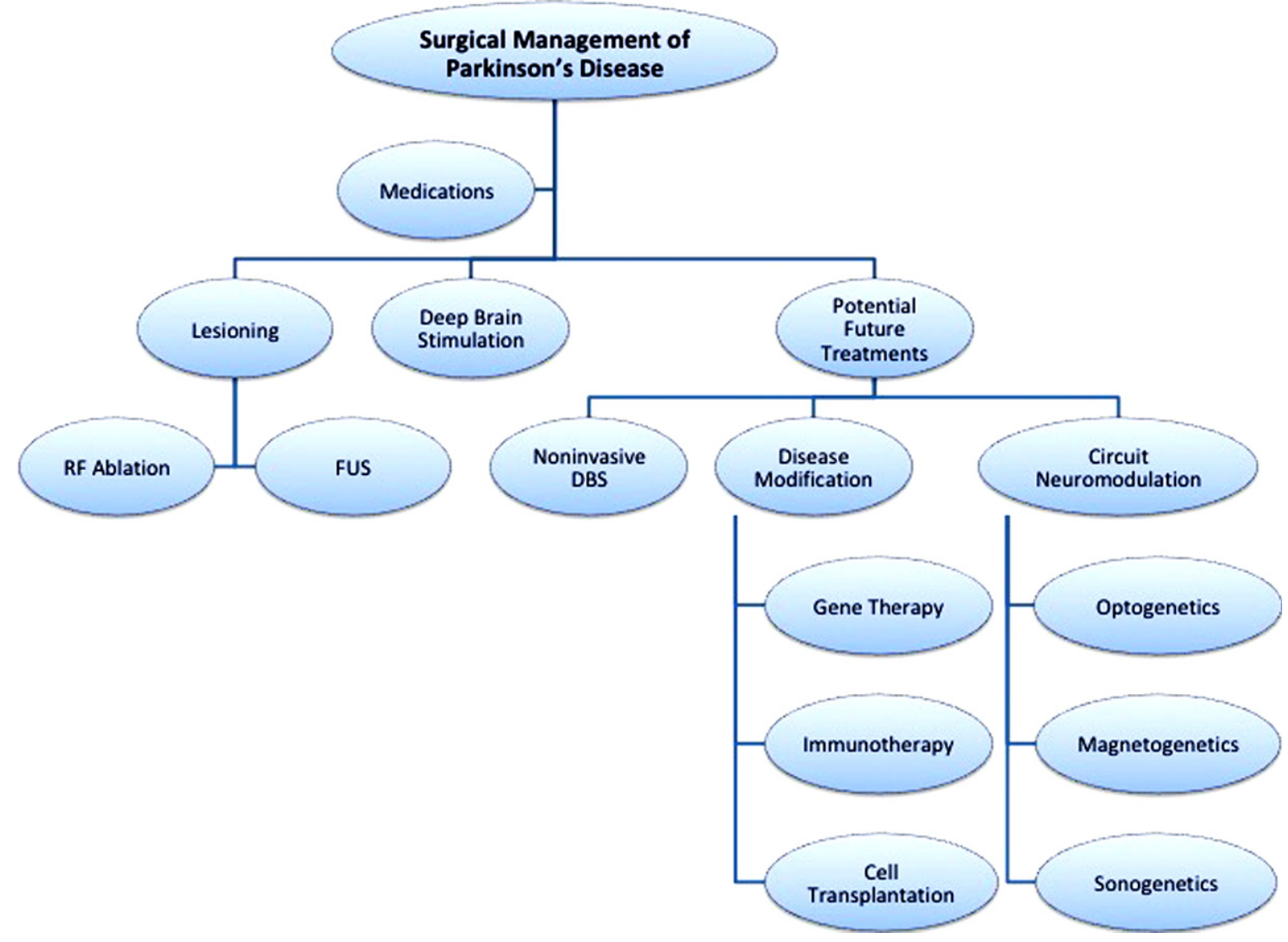

Fig. 1. Current and potential novel treatments of Parkinson's disease. RF ablation, radiofrequency ablation; FUS, focused ultrasound; DBS, deep rain stimulation.

early, but to date, immunotherapy trials have shown safety and feasibility but with limited clinical efficacy [16].

Loss of dopaminergic neurons within the substantia nigra is one of the defining characteristics in PD. Consequently, the landmark transplantation of fetal dopamine-containing neurons into a rodent model and subsequent improvement of motor function suggested that cell transplantation would offer a potentially exciting novel treatment $[17,18]$. Human clinical trials at various stages of development are utilizing autologous and nonautologous cells, including human embryonic stem cells and induced pluripotent stem cells [19]. Regrettably, like the gene therapy and immunotherapy clinical trials, there has yet to be clear clinical success. One of the additional impediments to this treatment is the potential ethical consideration that is associated with the use of stem cells. This can be avoided by using a variety of other types of stem cells including induced pluripotent stem cells, and a number of trials are now considering this novel approach [20].

While promising, these disease-modifying treatments have not shown sufficient clinical utility to date. It is important to identify the potential sources of failure. Clinical trials have shown that there can be a significant placebo response in studies of PD surgery [21]. Moreover, establishing useful measures of success has been challenging. For example, there are limited validated biomarkers of disease progression. Success in animal models has been less predictive than one would like. Further work needs to be done to account for differences in animal versus human biology. Subsequently, there have been a number of new techniques that have been developed to help better understand the disease process and potentially be used as treatment modalities in the future. Currently, optogenetics, magnetogenetics, and sonogenetics have been proposed as potential gene-altering and circuit modulation treatments [22]. 
Optogenetics has revolutionized the field of neuroscience by enabling scientists to determine causal relationships between neuronal activity and behavior. In brief, optogenetics enables the manipulation of neuronal activity using microbial membrane opsins that are sensitive to light (such as channelrhodopsin or halorhodopsin). While DBS affects multiple nuclei simultaneously, optogenetics can potentially excite or inhibit specific cell-types within a focal region of nuclei, which would enable more precise neuromodulation. This technique is temporally and spatially very precise, but requires invasive surgery and is limited by the opacity of the brain and is reliant on a light source [23].

Magnetogenetics utilizes a similar principal to optogenetics, but relies on the activation of neurons via magnetic fields that pass through the tissue to activate genetically expressed magnetoreceptors or nanoparticles. The challenge with magnetogenetics is that there is less temporal precision, as the activation/ deactivation kinetics are slower than physiological neural activity. The receptors may also be susceptible to endogenous stimuli like temperature and $\mathrm{pH}$ changes, potentially limiting the applicability [22].

Sonogenetics is a technique based on focused ultrasound with the potential for spatial, cell-type and temporal control without invasive surgery. Potential applications of this technique include opening the blood brain barrier with focused ultrasound, followed by viral vector gene delivery to specific cell types, and chemogenetic receptors or mechanoreceptors at the target neurons [24]. While this treatment strategy may have better temporal resolution than magnetogenetic approaches, it still does not have the same temporal specificity as optogenetics or deep brain stimulation.

These treatment modalities alone or in combination have the potential to be transformative in the field of neuromodulation for PD. Overcoming the barriers to cell-specific manipulation on a temporally precise timescale remains the ultimate goal. This could have major implications for not only motor symptoms but also the entire disease process as a whole.

The early treatments of PD and the field of functional neurosurgery were based on lesioning procedures; however, the advent of a reversible electrical stimulation treatment modality has dominated this field over the past four decades. While surgical treatments have been effective for a select population of PD patients, it is of paramount importance to be able to treat the entire PD population. PD is not a homogenous disease and can vary significantly between individuals. With these new techniques, it will also be important to individualize treatments based upon the needs of the patients. Current treatments are already multidisciplinary, including medical and surgical management; however, future treatments may involve even more interventions. These future interventions may involve integrating cell-specific and network-specific treatments. The ultimate goal of treatment is to not only treat motor symptoms but to slow down and cure the disease process. In order to achieve this Holy Grail, the field will have to move beyond deep brain stimulation. Instead, the future of PD treatment may lie in invasive and/or noninvasive neuromodulatory approaches that modify the disease process.

\section{ACKNOWLEDGMENTS}

Dr. Lozano is the R.R. Tasker Chair in Functional Neurosurgery at University Health Network and Tier 1 Canada Research Chair in Neuroscience.

\section{CONFLICT OF INTEREST}

Dr. Lozano is a consultant to Medtronic, St. Jude. and Boston Scientific. Dr. Lee has no conflict of interest to report.

\section{REFERENCES}

[1] Lozano CS, Tam J, Lozano AM (2018) The changing landscape of surgery for Parkinson's disease. Mov Disord 33, 36-47.

[2] Narabayashi H, Okuma T (1953) Procaine-oil blocking of the globus pallidus for the treatment of rigidity and tremor of parkinsonism. Proc Japan Acad 29, 134-137.

[3] Hassler R, Riechert T (1954) [Indications and localization of stereotactic brain operations]. Nervenarzt 25, 441-447.

[4] Follett KA, Weaver FM, Stern M, Hur K, Harris CL, Luo P, Marks WJ, Jr., Rothlind J, Sagher O, Moy C, Pahwa R, Burchiel K, Hogarth P, Lai EC, Duda JE, Holloway K, Samii A, Horn S, Bronstein JM, Stoner G, Starr PA, Simpson R, Baltuch G, De Salles A, Huang GD, Reda DJ, CSP 468 Study Group (2010) Pallidal versus subthalamic deepbrain stimulation for Parkinson's disease. N Engl J Med 362, 2077-2091.

[5] Collomb-Clerc A, Welter ML (2015) Effects of deep brain stimulation on balance and gait in patients with Parkinson's disease: A systematic neurophysiological review. Neurophysiol Clin 45, 371-388.

[6] Stefani A, Lozano AM, Peppe A, Stanzione P, Galati S, Tropepi D, Pierantozzi M, Brusa L, Scarnati E, Mazzone P (2007) Bilateral deep brain stimulation of the pedunculopontine and subthalamic nuclei in severe Parkinson's disease. Brain 130, 1596-1607.

[7] Gratwicke J, Zrinzo L, Kahan J, Peters A, Beigi M, Akram H, Hyam J, Oswal A, Day B, Mancini L, Thornton J, Yousry 
T, Limousin P, Hariz M, Jahanshahi M, Foltynie T (2018) Bilateral deep brain stimulation of the nucleus basalis of Meynert for Parkinson disease dementia: A randomized clinical trial. JAMA Neurol 75, 169-178.

[8] Schupbach WMM, Chabardes S, Matthies C, Pollo C, Steigerwald F, Timmermann L, Visser Vandewalle V, Volkmann J, Schuurman PR (2017) Directional leads for deep brain stimulation: Opportunities and challenges. Mov Disord 32, 1371-1375.

[9] Wagle Shukla A, Zeilman P, Fernandez H, Bajwa JA, Mehanna R (2017) DBS programming: An evolving approach for patients with Parkinson's disease. Parkinsons Dis 2017, 8492619.

[10] Swann NC, de Hemptinne C, Thompson MC, Miocinovic S, Miller AM, Gilron R, Ostrem JL, Chizeck HJ, Starr PA (2018) Adaptive deep brain stimulation for Parkinson's disease using motor cortex sensing. J Neural Eng 15, 046006.

[11] Rosin B, Slovik M, Mitelman R, Rivlin-Etzion M, Haber SN, Israel Z, Vaadia E, Bergman H (2011) Closed-loop deep brain stimulation is superior in ameliorating parkinsonism. Neuron 72, 370-384.

[12] Martinez-Fernandez R, Rodriguez-Rojas R, Del Alamo M, Hernandez-Fernandez F, Pineda-Pardo JA, Dileone M, Alonso-Frech F, Foffani G, Obeso I, Gasca-Salas C, de Luis-Pastor E, Vela L, Obeso JA (2018) Focused ultrasound subthalamotomy in patients with asymmetric Parkinson's disease: A pilot study. Lancet Neurol 17, 54-63.

[13] Bond AE, Shah BB, Huss DS, Dallapiazza RF, Warren A, Harrison MB, Sperling SA, Wang XQ, Gwinn R, Witt J, Ro S, Elias WJ (2017) Safety and efficacy of focused ultrasound thalamotomy for patients with medication-refractory, tremor-dominant Parkinson disease: A randomized clinical trial. JAMA Neurol 74, 1412-1418.

[14] Grossman N, Bono D, Dedic N, Kodandaramaiah SB, Rudenko A, Suk HJ, Cassara AM, Neufeld E, Kuster N, Tsai LH, Pascual-Leone A, Boyden ES (2017) Noninvasive deep brain stimulation via temporally interfering electric fields. Cell 169, 1029-1041, e1016.
[15] Bartus RT, Weinberg MS, Samulski RJ (2014) Parkinson's disease gene therapy: Success by design meets failure by efficacy. Mol Ther 22, 487-497.

[16] George S, Brundin P (2015) Immunotherapy in Parkinson's disease: Micromanaging alpha-synuclein aggregation. J Parkinsons Dis 5, 413-424.

[17] Bjorklund A, Stenevi U (1979) Reconstruction of the nigrostriatal dopamine pathway by intracerebral nigral transplants. Brain Res 177, 555-560.

[18] Perlow MJ, Freed WJ, Hoffer BJ, Seiger A, Olson L, Wyatt RJ (1979) Brain grafts reduce motor abnormalities produced by destruction of nigrostriatal dopamine system. Science 204, 643-647.

[19] Yasuhara T, Kameda M, Sasaki T, Tajiri N, Date I (2017) Cell therapy for Parkinson's disease. Cell Transplant 26, 1551-1559.

[20] Li W, Chen S, Li JY (2015) Human induced pluripotent stem cells in Parkinson's disease: A novel cell source of cell therapy and disease modeling. Prog Neurobiol 134, 161177.

[21] Keitel A, Wojtecki L, Hirschmann J, Hartmann CJ, Ferrea S, Sudmeyer M, Schnitzler A (2013) Motor and cognitive placebo-/nocebo-responses in Parkinson's disease patients with deep brain stimulation. Behav Brain Res 250, 199-205.

[22] Rajasethupathy P, Ferenczi E, Deisseroth K (2016) Targeting neural circuits. Cell 165, 524-534.

[23] Deisseroth K, Feng G, Majewska AK, Miesenbock G, Ting A, Schnitzer MJ (2006) Next-generation optical technologies for illuminating genetically targeted brain circuits. J Neurosci 26, 10380-10386.

[24] Szablowski JO, Lee-Gosselin A, Lue B, Malounda D, Shapiro MG (2018) Acoustically targeted chemogenetics for the non-invasive control of neural circuits. Nat Biomed Eng 2, 475-484. 